\title{
Avaliação da saúde bucal de pacientes portadores de diabetes mellitus tipo II atendidos em uma Unidade Básica de Saúde no Estado do Amazonas
}

\author{
Evaluation of the oral health of type II diabetes mellitus patients in a Primary Health Care \\ Unit in the State of Amazonas
}

\section{Evaluación de la salud bucal de pacientes con diabetes mellitus tipo II atendidos en una Unidad de Atención Primaria en el Estado de Amazonas}

Maria das Graças Silva Sarmento ${ }^{1}$, Michelli Domingos da Silva² , James Lee Crainey.

\begin{abstract}
RESUMO
Objetivo: Este relato de experiência tem o objetivo de avaliar e descrever a luz da literatura sobre a saúde bucal dos pacientes portadores de diabetes mellitus tipo II atendidos na Unidade Básica de Saúde no Estado do Amazonas. Relato de experiência: Trata-se de um estudo descritivo, do tipo relato de experiência, acerca do período em atendimento de saúde bucal em portadores de diabetes mellitus, em uma unidade básica de saúde no Estado do Amazonas no período de janeiro a dezembro de 2019. Foi vivenciado que existe um alto índice de pessoas com dentes permanentes cariados, perdidos e obturados (CPOD), diabéticos através do atendimento especializado na UBS. A UBS conta hoje com equipes de saúde especializadas, e visa controlar a glicemia do paciente durante o tratamento odontológico. Os auxiliares de saúde bucal e os odontólogos, podem contribuir para melhora da qualidade de vida desses pacientes, ficando responsável pela realização de uma boa higiene oral. Considerações finais: Com relação as condições de saúde bucal, foi confirmado um auto índice de diabéticos que possui o índice de dentes cariados e obturados mais elevado, necessita de maior intervenção periodontal e classicamente evidenciou-se maiores problemas periodontias neste grupo.
\end{abstract}

Palavras-chave: Odontologia, Diabetes mellitus tipo II, Saúde pública.

\section{ABSTRACT}

Objective: This experience report aims to evaluate and describe in the light of literature the oral health of patients with type II diabetes mellitus seen in a Basic Health Unit in the State of Amazonas. Experience report: This is a descriptive study, of the experience report type, about the period in oral health care in patients with diabetes mellitus, in a basic health unit in the State of Amazonas in the period from January to December 2019. It was experienced that there is a high rate of people with decayed, lost and filled permanent teeth (CPOD), diabetics through specialized care in the UBS. The UBS now has specialized health teams, and aims to control the patient's blood sugar during dental treatment. Oral health assistants and dentists can contribute to the improvement of the quality of life of these patients, being responsible for the accomplishment of a good oral hygiene. Final considerations: Regarding the oral health conditions, it was confirmed that diabetics have the highest index of decayed and filled teeth, require greater periodontal intervention and classically showed greater periodontal problems in this group.

Key words: Dentistry, Type II diabetes mellitus, Public health.

\section{RESUMEN}

Objetivo: Este informe de experiencia tiene como objetivo evaluar y describir a la luz de la literatura la salud bucal de los pacientes con diabetes mellitus tipo II atendidos en una Unidad Básica de Salud del Estado de Amazonas. Informe de experiencia: Se trata de un estudio descriptivo, de tipo informe de experiencia, sobre el período en la atención a la salud bucal en pacientes con diabetes mellitus, en una unidad básica de salud del Estado de Amazonas en el período de enero a diciembre de 2019. Se experimentó que hay un alto índice de personas con dientes permanentes cariados, perdidos y empastados (CPOD), diabéticos a través de la atención especializada en UBS. La UBS cuenta ahora con equipos sanitarios especializados, y su objetivo es controlar la glucemia del paciente durante el tratamiento dental. Los asistentes de salud bucodental y los dentistas pueden contribuir a mejorar la calidad de vida de estos pacientes, siendo responsables de realizar una buena higiene bucodental. Consideraciones finales: En cuanto a las condiciones de salud bucal, se confirmó que los diabéticos tienen un alto índice de dientes cariados y obturados, requiriendo una mayor intervención periodontal y evidenciando clásicamente mayores problemas periodontales en este grupo.

Palabras clave: Odontología, Diabetes mellitus tipo II, Salud pública.

\footnotetext{
${ }^{1}$ Fundação Oswaldo Cruz. Instituto Leônidas e Maria Deane (ILMD), Manaus - AM.

2 Universidad de Ciencias Empresariales y Sociales (UCES), Buenos Aires - AR.

*E-mail: michelliclarinha@hotmail.com
} 


\section{INTRODUÇÃO}

Em 2016, Margaret Chan, diretora geral da Organização Mundial de Saúde (OMS), lança o primeiro relatório global sobre diabetes usando as seguintes palavras em seu prefácio: temos um trabalho enorme para ser desenvolvido juntos com a população, sinalizando a preocupação dos líderes mundiais com relação a esta patologia que além de mutiladora e mortal, prejudica em nível global a economia de todos os países (WHO, 2016).

No Brasil, no ano de 2015 houve uma predominância de 14,3 milhões de pessoas diabéticas, estima-se que esta população chegará a 23,2 milhões de pessoas em 2030 (CHO NH, et al., 2018; ASSIS MMA, et al., 2012). O perfil dos pacientes acometidos com diabetes estudado por Artilheiro MMVSA, et al. (2014), ao analisar as internações desses pacientes no Sistema Único de Saúde (SUS), baseou-se no município de Joinville/SC/Brasil afirma ainda que $5,2 \%$ das mortes é devido a essa patologia, afetando de sobremodo os grupos vulneráveis, em especial, pessoas de baixa renda e idosas.

Diabetes, é um distúrbio metabólico relacionado à falta de secreção de insulina ou a sua não efetividade no metabolismo de carboidratos, proteínas e lipídios. Se não devidamente controlada o portador sofre degeneração física através dos problemas renais, circulatórios e metabólicos até a morte (OMS, 2016; IFD, 2017).

Sabe-se que o pâncreas é uma glândula com funções importantes para o sistema endócrino, por vários motivos como falha na secreção das células $\beta$, tumores malignos, inflamação do órgão dentre outras causas deixa de excretar o hormônio; ou o próprio organismo deixa de estar sensível a esta substância, resultando na resistência insulínica, seja dado a algum erro na produção de receptores que se acoplam à insulina ou na transdução do sinal para as enzimas responsáveis pela captação da glicose, provocando um aumento dos níveis de açúcar na corrente sanguínea e todo um descontrole metabólico (NELSON DL e COX MM, 2014).

A captação de glicose pelos miócitos e adipócitos é mediada pelo transportador de glicose GLUT4 (Transportador de glicose tipo IV). Entre as refeições, alguns GLUT4 estão presentes na membrana plasmática, porém, a maioria encontra-se sequestrada das membranas em pequenas vesículas intracelulares. A insulina, liberada pelo pâncreas (células $\beta$ das ilhas de Langherhans), em resposta a alta concentração de glicose sanguínea, desencadeia o movimento dessas vesículas intracelulares à membrana plasmática, onde elas se fundem, expondo assim os transportadores na superfície externa da célula (NELSON DL e COX MM, 2014).

Quando os níveis de glicose sanguínea retornam ao normal, a liberação de insulina torna-se lenta, a maioria das moléculas de GLUT4 é removida da membrana plasmática e armazenada em vesícula. Caso não haja uma melhora na mudança do comportamento da sociedade, envolvendo novos hábitos alimentares, modo de vida ou modelo de produção, os países arcarão com um alto custo para o tratamento desses pacientes, desde absenteísmo no sistema de trabalho e custos com auxílios a medicamentos e recursos humanos no atendimento à saúde destes (FRANÇA DJT, et al., 2014).

Objetivo deste estudo é avaliar e relatar uma experiência sobre a saúde bucal dos pacientes portadores de diabetes mellitus tipo II atendidos em uma Unidade Básica de Saúde (UBS) no Estado do Amazonas.

\section{RELATO DE EXPERIÊNCIA}

Trata-se de um estudo descritivo, do tipo relato de experiência, no do período em atendimento de saúde bucal em portadores de diabetes mellitus, em uma unidade básica de saúde no Estado do Amazonas no período de janeiro a dezembro de 2019. De acordo com o censo do Instituto Brasileiro de Geografia e Estatística (IBGE), o bairro tem a população de 7.632 habitantes em 2020.

A UBS começou a funcionar a partir das $7 \mathrm{~h}$ da manhã e seu fechamento se dá às $19 \mathrm{~h}$. Uma das políticas adotadas pela direção da UBS, é que todas as pessoas que possuem as fichas de atendimento, irão ser atendidas até o encerramento outros entram no sistema de agendamentos para uma data próxima no qual foi atendido naquele dia. 
Na UBS, são oferecidos ao cliente diversos serviços, como consulta médica com clínico geral e pediatria, consulta de enfermagem em atenção primária como: preventivo, teste rápido, pré-natal e planejamento familiar, administração de medicamentos, aferição de pressão arterial, atividades de promoção e prevenção à saúde, acompanhamento de programas sociais, curativo, dispensação de medicamentos (Farmácia Gratuita), imunização, nebulização e serviço odontológico.

O tratamento é gratuito, oferecido apenas na rede Sistema Único de Saúde (SUS), tendo duração que ocorre de paciente para paciente podendo ser dias ou meses. Há casos em que o tratamento precisa ser estendido por mais tempo, a depender da avaliação clínica e de resultados de exames laboratoriais para possíveis cirurgias. É comum que após as primeiras semanas de tratamento, o paciente observe melhora na sua mastigação e até na sua halitose. No entanto, isto não quer dizer que o procedimento odontológico seja finalizado de imediato, no entanto para obter sucesso no sorriso tem que fazer o tratamento correto.

Durante as consultas de odontologia, foram verificadas a glicose pós-prandial, visto que nem todos os pacientes apresentaram hemoglobina glicosada ou glicose jejum, pois o laboratório que realiza esses exames não estava disponível para todos os pacientes seja por falta de reagentes para realizar os exames especificados. No geral, os atendimentos com os pacientes diabéticos são tranquilos, pois entendemos que devem ser realizados os sinais vitais e Hemoglobina Glicosilada Teste (HGT), para poder dá continuidade ao seu tratamento dentário. Analisando os pacientes com ou sem diabetes, houve uma diferença significativa, vista nitidamente a necessidade de um acompanhamento mais intenso aos pacientes diabéticos, pois houve indivíduos com glicose pós-prandial tiveram um aumento da glicemia alegando que estavam adequadamente tomando seus medicamentos de controle.

O acolhimento na UBS foi um dos processos discutidos e implantado na unidade, tem como objetivo primordial da voz a população. Enxergou-se essa necessidade, visto que antes a escuta era concretizada em consultas agendadas. Além disso, quando se concentrava este acolhimento só nas consultas, nunca dava tempo de fazer todo o procedimento odontológico necessário e as vezes era insuficiência de equipe de saúde para atender a demanda geram sobrecarga de trabalho.

Porém hoje em dia a UBS, oferece um atendimento diferenciado por ter quatro equipes de saúde que estão voltadas para o acolhimento de forma organizada, lidando com as situações de demanda espontânea, sem que esta ocupe o tempo reservado e necessário para a construção do cuidado longitudinal da demanda programada na UBS, contribuindo assim para o estabelecimento de vínculo entre o profissional e o paciente.

Uma das experiências corriqueiras nas UBSs, ocorreram a partir do argumento apresentado as habilidades da saúde bucal, devem ser ofertadas através da demanda expoente sem o estigma de que os usuários estejam insatisfeitos com o atendimento, esses novos olhares e concepções vão surgir sempre quando lidamos com pessoas. Estes novos fluxos das UBSs e abordagens, compondo novas práticas em saúde bucal que potencialmente retribuíssem à inovação almejada pela Política Nacional de Saúde Bucal. Entretanto, essas experiências de novas arrumações para que a clínica de saúde bucal vem trazendo algumas queixas, como centro da atuação e do trabalho desenvolvido nesta comunidade.

\section{DISCUSSÃO}

Na região do Viver Melhor, o público que comparecem aos postos de saúde é marcadamente feminino, incluso o grupo de pacientes diabéticos o que vai de encontro com a maioria dos dados em que o sexo masculino é preponderante a nível nacional e de acordo com estudos já realizados em Joinville/SC e Manaus/AM (ARTILHEIRO MMVSA, et al., 2014; BRASIL, 2017).

Para uma patologia que acometia pessoas mais velhas, nota-se uma modificação neste quadro, pessoas mais jovens estão sendo atingidas, possivelmente devido a mudança na base alimentar da população, em especial a deste estudo, pelo consumo de mais carboidratos e poucos alimentos saudáveis, o que corrobora com a previsão de órgãos pesquisadores na área (WHO, 2014; $\mathrm{CHO} \mathrm{NH}$, et al., 2018).

Apesar da frequência de visitas ao médico, $64,3 \%$ dos diabéticos não realizam atividades físicas. No presente estudo verificou-se que verduras, legumes e peixe são os alimentos mais utilizados pelo o grupo de 
pacientes diabéticos, todavia há a necessidade de reforço para que haja uma mudança efetiva e permanente de hábitos, e todos os programas de prevenção pode eficazmente trabalhar nesse foco baseando-se nas diferenças econômicas, sociais e culturais, utilizando por exemplo uma estratégia de ativação da intenção juntamente com a estratégia motivacional tradicional integrando usuário e profissional de saúde (SILVA JVM, et al., 2015).

Especificamente para odontologia, Rosendo RA e Freitas CHSM, (2012), relata que as desigualdades sociais implicam nas diferenças nos padrões de doenças e de utilização de serviços e o grande desafio é atender essas necessidades de forma holística e por meio de programas de orientação e acompanhamento almejando a adesão do paciente ao tratamento proposto com consequente controle da patologia.

Ao se falar de saúde oral, os índices de dentes permanentes cariados, perdidos e obturados (CPOD) foram diferentes entre os grupos, sendo que o grupo de diabéticos possuía uma média mais elevada que do grupo controle, o que corrobora com alguns estudos na área, contudo Amaral FM, et al. (2006), não encontrou esses dados em sua amostra, sugerindo que os diabéticos apresentam menos cáries que o grupo controle, alegando que provavelmente a duração da patologia poderia contribuir para a ocorrência de cárie, e o menor consumo de sacarose poderia explicar sua menor frequência nesses pacientes.

Uma das explicações para esse alto índice, leva em conta que o fluido salivar possui alto teor de açúcar, isto é, a hiperglicemia era responsável pelo o alto nível de carboidratos na saliva e fluido gengival, e como é um dos fatores mais preponderantes no complexo multifatorial da formação de cárie, seria uma das explicações para o alto índice de bactérias orais iniciadoras da desmineralização dentária (SOUZA MGM, et al., 2011; KAMPOO K, et al., 2014).

Outro fator a se levar em questão é o baixo fluxo salivar que estes pacientes possuem, a xerostomia, muito devido a patologia que apresenta essa sintomatologia, como dado aos medicamentos utilizados para 0 controle metabólico, na tentativa de diminuir a produção de AGEs e fatores pro inflamatórios da patologia (KAMPOO K, et al., 2014).

Quando se fala em escolaridade, quanto mais o indivíduo é escolarizado espera-se que possui mais entendimento de sua situação física e o autocuidado estaria implícito neste conceito o que poderia tornar sua condição de saúde melhor, com consequentemente aumento de sua qualidade de vida, pois as desigualdades sociais geralmente modificam as diferenças nos padrões das doenças e na utilização dos serviços (ROSENDO RA, FREITAS CHSM 2012; ARTILHEIRO MMVSA et al., 2014).

Poudel P, et al. (2018), em sua revisão sistemática com pacientes diabéticos, concluiu que muitos não possuíam conhecimento sobre saúde oral e isso se refletia em péssimos hábitos de saúde oral. Quando se analisa o fator visitas ao dentista, é perceptível - há mais de 1 ano sem consulta odontológica - fornece subsídios de que há a necessidade não apenas para o grupo de diabéticos como de controle de fortalecimento do papel da odontologia na vida dos usuários e de integrá-la no sistema de cuidados (SERVERSON MJ, et al., 2014).

Ao analisar o uso de prótese dentária, da amostra de 110 indivíduos (grupo controle e diabético), 49,1\% a utilizam, o que significa uma falha no acesso da população à odontologia interferindo em sua qualidade de vida, sendo necessário ações mais pragmáticas não apenas nas ações coletivas educativas, mas preventivas e curativas Oliveira EJP, et al., (2018), o que corrobora com Silva AM, et al., (2010) ao afirmar que apenas $27,3 \%$ dos diabéticos estão em tratamento odontológico na rede básica, e 3,6\% recebiam atendimento especializado.

Com relação as condições de saúde bucal, foi confirmado um auto índice de diabéticos que possui o índice de dentes cariados e obturados mais elevado, necessita de maior intervenção periodontal e classicamente evidenciou-se maiores problemas periodontias neste grupo. Necessária uma maior efetividade dos serviços odontológico, com maior atuação do cirurgião-dentista na equipe multiprofissional, para que o atributo da integralidade esteja presente não apenas na vida dos usuários diabéticos, mas da população atendida. Todavia essa efetividade deve-se pautar em novas abordagens ao usuário dentro de programas e ações já desenvolvidas para real efetividade dessas ações para que o paciente seja o regente de sua própria saúde. 


\section{REFERÊNCIAS}

1. AMARAL FM, et al. Estudo da frequência de cárie e fatores associados no diabetes mellitus tipo 1. Arquivos Brasileiros de Endocrinologia \& Metabologia, 2006; 50(3): 515-522.

2. ARTILHEIRO MMVSA, et al. Quem são e como são tratados pacientes que internam por diabetes mellitus no SUS? Saúde Debate, 2014; 38(101): 210-224.

3. ASSIS MMA, et al. Acesso aos serviços de saúde: uma possibilidade a ser construída na prática. Ciência \& saúde coletiva, 2003; 8(3): 815-823.

4. BRASIL. 2018. In. Departamento de Vigilância de Doenças e Agravos não Transmissíveis e Promoção da Saúde. Vigitel Brasil 2017: vigilância de fatores de risco e proteção para doenças crônicas por inquérito telefônico: estimativas sobre frequência e distribuição sociodemográfica de fatores de risco e proteção para doenças crônicas nas capitais dos 26 estados brasileiros e no Distrito Federal em 2017 / Ministério da Saúde. Disponível em: https://bvsms.saude.gov.br/bvs/publicacoes/vigitel_brasil_2017_vigilancia_fatores_riscos.pdf. Acesso em: 02 de jan de 2017.

5. CAMELO MS, et al. Acolhimento na atenção primária à saúde na ótica de enfermeiros. Acta Paulista de Enfermagem, 2016; 29(4): 463-468.

6. CARVALHO CHR. Desafios da mobilidade urbana no Brasil. Texto para Discussão, 2016.

7. FRANCA DJT, et al. As contribuições do cuidado ao idoso no programa de HIPERDIA, para a formação profissional. Revista Kairós: Gerontologia, 2014; 17(2): 315-327.

8. $\mathrm{CHO} \mathrm{NH}$, et al. IDF diabetes atlas: Global estimates of diabetes prevalence for 2017 and projections for 2045. Diabetes Res Clin Pract 2018; 138: 271-81.

9. KAMPOO K, et al. Oral Bacterial Communities in Individuals with Type 2 Diabetes Who Live in Southern Thailand. Applied and Environmental Microbiology, 2016; 80(2): 662-671.

10. LIMA AIC, et al. Contribuições da equipe de saúde bucal para o acolhimento em uma Unidade de Saúde da Família: Relato de Experiência. Tempus Actas de Saúde Coletiva, 2019; 13(3): 201-209.

11. NELSON DL, COX MM. LEHNINGER Princípios de Bioquímica. 6aㅡ. Ed. São Paulo: Artmed, 2014.

12. OLIVEIRA EJP, et al. Qualidade de vida e condições de saúde bucal de hipertensos e diabéticos em um município do Sudeste Brasileiro. Ciência \& Saúde Coletiva, 2018; 23: 763-772.

13. POUDEL $P$, et al. Oral health knowledge, attitudes and care practices of people with diabetes: a systematic review. BMC public health, 2018; 18(1): 1-12.

14. ROSENDO RA, FREITAS CHSM. Diabetes Melito: dificuldades de acesso e adesão de pacientes ao Programa de Saúde da Família. Rev Bras Ciênc Saúde. 2012;16(1):13-20.

15. SEVERSON MJ, et al. Diabetes and Oral Health. ADHA Acess Diabetes, 2014; 23-24.

16. SILVA AM, et al. A integralidade da atenção em diabéticos com doença periodontal. Ciência \& Saúde Coletiva, 2010; 15(4): 2197-2206.

17. SILVA JVM, et al. Avaliação do programa de hipertensão arterial e diabetes mellitus na visão dos usuários. Rev. Bras. Enferm, 2015; 68(4): 626-632.

18. SOUSA MGM, et al. Clinical study of the oral manifestations and related factors in type 2 diabetics patients. Brazilian journal of otorhinolaryngology, 2011; 77(2): 145-152.

19. WORLD HEALTH ORGANIZATION (WHO). Diagnostic criteria and classification of hyperglycemia first detected in pregnancy: a World Health Organization Guideline. Diabetes research and clinical practice, 2014; 103: 341-363 